\title{
Therapeutic effects of tofacitinib on pristane-induced murine lupus
}

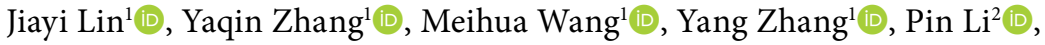 \\ Yingping $\mathrm{Cao}^{1}\left(\mathbb{D}\right.$, Xuwei Yang ${ }^{2}$ (I) \\ ${ }^{1}$ Department of Laboratory Medicine, Fujian Medical University Union Hospital, Fuzhou, China \\ ${ }^{2}$ Department of Rheumatology, Fujian Medical University Union Hospital, Fuzhou, China
}

\begin{abstract}
Objectives: This study aims to investigate the effectiveness of tofacitinib, a Janus kinase (JAK) $1 /$ JAK3 inhibitor, in treating murine lupus, and also explore 12 related genes downstream of JAK-signal transducer and activator of transcription (STAT) signaling pathways to find the underlying mechanism.

Materials and methods: This study was conducted between July 2017 and January 2020. Fifty-seven female BALB/c mice (aging 8 to 10 weeks old; weighing 18 to $20 \mathrm{~g}$ ) were assigned to a saline control (SC) group and a pristane-induced lupus group. The latter included four groups, namely, pristane control (PC), tofacitinib (T), methylprednisolone (MP), and tofacitinib plus methylprednisolone (T+MP). Animal models of lupus were induced with pristane, whereas SC mice were treated with normal saline. From the $22^{\text {nd }}$ week after induction, each group was given the aforementioned corresponding intervention for 11 weeks. The following variables were tested: serum concentrations of anti-double-stranded deoxyribonucleic acid (anti-dsDNA), interleukin 6 (IL-6), and interferon gamma (IFN- $\gamma$ ); number of regulatory T (Treg) cells; messenger ribonucleic acid levels of forkhead box $\mathrm{P} 3$ and 12 related genes downstream of JAK-STAT pathway; and renal impairment.

Results: Red swollen joints and proteinuria were first observed in PC after the $12^{\text {th }}$ week. After treatment, T, MP, and T+MP showed relieved red swollen joints and splenomegaly, as well as decreased urine protein, anti-dsDNA, IL-6, IFN- $\gamma$, Treg cells, pathological scores, and hyperplasia of mesangial matrix in glomeruli compared with PC. The IFN regulatory factor 7 level was higher in T+MP $(p<0.01)$ and slightly higher in T $(p>0.05)$ and MP ( $p>0.05)$ than in PC after treatment. The expression of suppressor of cytokine signaling (SOCS) 1 was lower in $T(p>0.05), T+M P(p<0.01)$, and MP $(p>0.05)$ than in PC. The SOCS3 level was higher in T $(p>0.05)$ and T+MP $(p<0.01)$ and lower in MP $(p>0.05)$ than in PC.

Conclusion: Tofacitinib can ameliorate glomerulonephritis and arthritis in a pristane-induced murine model of lupus. SOCS3 gene may be involved in the therapeutic mechanism of tofacitinib.

Keywords: Janus kinase, signal transducer and activator of transcription, systemic lupus erythematosus, tofacitinib.
\end{abstract}

Systemic lupus erythematosus (SLE) is a chronic autoimmune disease (AID) that affects many organs. Approximately 40 to $60 \%$ of patients with SLE exhibit renal symptoms and lupus nephritis. ${ }^{1}$

The Janus kinase (JAK) signal transducer and activator of transcription (STAT) signaling pathway exhibits aberrant STAT signaling under inflammatory conditions and AIDs, including SLE. $^{2}$ In contrast to healthy controls, the peripheral blood mononuclear cells of patients with SLE show constitutive JAK1 and STAT2 phosphorylation even in the absence of disease activities. ${ }^{3}$ Many inflammatory cells and factors are related to the STAT signaling pathway. In AIDs such as SLE, the production of chronic

Received: June 15, 2020 Accepted: August 28, 2020 Published online: March 03, 2022

Correspondence: Xuwei Yang, MD. Department of Rheumatology, Fujian Medical University Union Hospital, 350001 Fuzhou, China. Tel: 008659183357896 e-mail: yangxwhk@126.com 
type 1 interferon (IFN) is partly perpetuated by the improper regulation or persistent stimulation of antigen-presenting cells, including plasmacytoid dendritic cells, immune complexes, and damageassociated molecular-pattern molecules. Persistent type 1 IFN exposure increases the effector function of $\mathrm{T}$ and $\mathrm{B}$ cells, leading to autoantibody production, and ultimately, AID. IFN-mediated priming can contribute to increased cell activation and responsiveness to inflammatory factors. The transcript and total STAT1 protein levels in T and $B$ cells significantly increase in SLE mouse models and patients with SLE, suggesting the involvement of augmented STAT1 signaling in response to IFNs. ${ }^{4} \mathrm{~T}$ cells from patients with SLE exhibit increased levels of total and phosphorylated STAT3. ${ }^{5}$ STAT3 is believed to play a crucial role in $\mathrm{T}$ cell differentiation into $\mathrm{T}$ helper 17 and $\mathrm{T}$ follicular helper cells, which aid B cells within the germinal center. ${ }^{6}$ Moreover, the increased STAT5 signaling in lupus $\mathrm{T}$ cells is related to changes in circulating cluster of differentiation $\left(C D 4^{+} \mathrm{T}\right.$ cell subsets and correlated with aggressive diseases. ${ }^{7}$

The JAK-STAT pathway plays a role in SLE pathogenesis. Inhibiting this pathway may be a promising therapeutic strategy for SLE. Following the success in treating rheumatoid arthritis (RA), ${ }^{8}$ tofacitinib, the first oral small molecule JAK inhibitor of the pathway, is extensively investigated due to its potential effects on AIDs.

In a phase II trial of non-severe SLE, baricitinib, a JAK1/JAK2 inhibitor, has led to significantly increased clinical responses, particularly improvements in skin and joint disease. ${ }^{9}$

In this study, we aimed to investigate the effectiveness of tofacitinib, a JAK1/JAK3 inhibitor, in treating murine lupus, and also explore 12 related genes downstream of JAK-STAT signaling pathways to find the underlying mechanism.

\section{MATERIALS AND METHODS}

This study was conducted at Fujian Medical University between July 2017 and January 2020. Fifty-seven female BALB/c mice (aging 8 to 10 weeks old; weighing 18 to $20 \mathrm{~g}$ ) were purchased from Shanghai Laboratory Animal Co. Ltd., and randomly assigned to a saline control group (SC; $\mathrm{n}=12$ ) or a pristane-induced group $(n=45)$. The latter was divided into a pristane control group ( $\mathrm{PC} ; \mathrm{n}=12)$ and three therapy groups: tofacitinib (Pfizer, Manhattan, New York City, New York, USA; 10 mg/kg; $\mathrm{n}=12$ ), methylprednisolone (Pfizer, Manhattan, New York City, New York, USA; 4 mg/kg; $\mathrm{n}=10$ ), and tofacitinib plus methylprednisolone (10 and $4 \mathrm{mg} / \mathrm{kg}$, respectively; $\mathrm{n}=11$ ). The three therapy groups are hereafter denoted as T, MP, and $\mathrm{T}+\mathrm{MP}$, respectively. Animal models of lupus were induced via intraperitoneal injection with $0.5 \mathrm{~mL}$ of pristane (Sigma-Aldrich, St. Louis, MO, USA) in PC, T, MP, and T+MP. Meanwhile, $\mathrm{SC}$ was treated with $0.5 \mathrm{~mL}$ of normal saline. ${ }^{10}$ From the $22^{\text {nd }}$ week, each group was given the aforementioned corresponding intervention measures (continuous oral gavage six days every week) for 11 weeks.

Body weight and leg joint diameter were measured every four weeks. At the end of the $32^{\text {nd }}$ week, the mice were sacrificed, and their spleen and kidney size and weight were measured.

Urine protein was tested every four weeks before and after injection. Urine protein was detected using test papers (Siemens, Henkestr, Erlangen, Germany). ${ }^{11}$

Kidney samples were harvested from the sacrificed mice and stained with hematoxylin-eosin (H-E) and periodic acid-Schiff (PAS). H-E-stained sections were graded semi-quantitatively for glomerular lesion score based on the following scheme: normal glomerulus, 0; focal, mild, or early proliferative, 1; multifocal proliferative with increased matrix and inflammatory cells, 2; diffuse proliferative, 3; and extensive sclerosis/ crescents, 4. Forty glomeruli were scored for each mouse, and the average was taken. ${ }^{11}$

Murine blood samples were obtained by inner cantheus vein bleeding. Serum levels of anti-double-stranded deoxyribonucleic acid (anti-dsDNA), interleukin-6 (IL-6), and IFN-gamma $(\gamma)$ were measured using a mouse anti-dsDNA immunoglobulin $\mathrm{G}$ antibody assay kit (Chrondrex Inc., Redmond, WA, USA), a mouse IL-6 DuoSet ${ }^{\circledR}$ enzyme-linked immunosorbent assay (ELISA) kit (R\&D Systems; Minneapolis, MN, USA), and a mouse IFN- $\gamma$ DuoSet ${ }^{\circledR}$ ELISA kit (R\&D Systems; Minneapolis, MN, USA). 
Splenic nucleated cells were prepared from the sacrificed mice by hemolytic method. The cells were then incubated with rat anti-mouse CD4-fluorescein isothiocyanate (FITC) monoclonal antibody (eBioscience ${ }^{\mathrm{TM}}$, San Diego, CA, USA) and rat anti-mouse CD25-phycoerythrin (PE) monoclonal antibody (BioLegend, San Diego, CA, USA). After washing, the samples were incubated with rat anti-mouse forkhead box P3 (Foxp3)allophycocyanin (APC) monoclonal antibody (eBioscience ${ }^{\mathrm{TM}}$, San Diego, CA, USA) at room temperature for $30 \mathrm{~min}$ and then analyzed by flow cytometry (FACSCanto ${ }^{\mathrm{TM}}$ II, BD Biosciences, San Jose, CA, USA).

Splenic total ribonucleic acid (RNA) was extracted with TRIzol ${ }^{\circledR}$ and reverse transcripted into complementary DNA with PrimeScript ${ }^{\mathrm{TM}} \mathrm{RT}$ reagent Kit with gDNA Eraser (TaKaRa, Kusatsu, Shiga, Japan). The relative expression levels of related messenger RNA (mRNA) were tested through quantitative polymerase chain reaction using TB Green ${ }^{\mathrm{TM}}$ Premix Ex Taq ${ }^{\mathrm{TM}}$ (TaKaRa, Kusatsu, Shiga, Japan). The adopted primers were listed in Table 1. Glyceraldehyde-3-phosphate dehydrogenase was used as the housekeeping gene. The relative gene expression was examined using the delta cycle threshold method.

\section{Statistical analysis}

Statistical analysis was performed using the IBM SPSS version 22.0 software (IBM Corp., Armonk, NY, USA). Data distribution was tested by Shapiro-Wilk method. Results were presented as the mean \pm standard deviation or number. Normally distributed data with homoscedasticity were analyzed using two-group t-test for independent samples or one-way analysis of variance. Non-normally distributed data and ordinal data were compared between the two groups with the Mann-Whitney $U$ test or Kruskal-Wallis $\mathrm{H}$ test. $\mathrm{P}<0.05$ was considered statistically significant.

\section{RESULTS}

Red swollen joints and proteinuria were first observed in PC after the $12^{\text {th }}$ week. Thus, the model of murine lupus was successfully established (Figure 1).

\begin{tabular}{|c|c|c|}
\hline Primer & Forward sequence & Reverse sequence \\
\hline Foxp3 & GTCTCACCTGCTCCTCAA & GCCTTGTATTCTTGCTGTCTC \\
\hline IRF1 & GAAGATAGCCGAAGACCTTATGA & GCTGTCAATCTCTGGTTCCT \\
\hline IRF3 & CTCCAACAGCCAGCCTAT & AGACTGAGCCTTGTAGAATAACC \\
\hline IRF4 & TGAAGAGGTAGGCTGAGGAA & AACGACTGAGGGCTTTGG \\
\hline IRF5 & GTGACAAGCAGCGTTTCTATAC & CCACTCCAGAACACCTTACA \\
\hline IRF7 & GCGAAGAGGCTGGAAGAC & TACACCTTATGCGGATCAACTG \\
\hline IRF8 & GCAGAAAGCCAAGCAGATG & GAGCAACAGTCAGCAGGTA \\
\hline IRF9 & CCTGCTTCTGTGATGGTTCT & GGTTGTGGAGGGTGAGATG \\
\hline SOCS1 & GGTTGTGGAGGGTGAGATG & ATGGAGAGGTAGGAGTGGAAT \\
\hline socs3 & CCGTTGTGAAGAGGCAGTA & TGGGACAGAGGGCATTTAAG \\
\hline SHC1 & GAGGCAGAGACAGGAGGAAT & AGGAGGCACTGAGCAAGAT \\
\hline PIAS1 & GTCACTGCCTCCTACAATGG & GGTTCTTCCTCCTCTTCATCTG \\
\hline PIAS2 & ACAGAGGTTGAAGACGATTGG & GCAGGTAGAACACAGCATCA \\
\hline GAPDH & AGGTCGGTGTGAACGGATTTG & TGTAGACCATGTAGTTGAGGTCA \\
\hline
\end{tabular}


Tofacitinib relieved red swollen joints and splenomegaly and decreased urine protein level in lupus mice. The leg joint diameters of mice with lupus decreased in T, MP, and T+MP with no intergroup differences (Figure 2a). Spleen weight and length (Figures $2 \mathrm{~b}$ and $\mathrm{c}$ ) were also lower in $\mathrm{T}$, MP, and T+MP than in PC. The proteinuria levels in lupus mice continuously increased after the pristane injection but decreased in $\mathrm{T}$, MP, and $\mathrm{T}+\mathrm{MP}$ after oral gavage (Figure $2 \mathrm{~d}$ ). In week 32 , urine protein was reduced in $\mathrm{T}, \mathrm{T}+\mathrm{MP}$, and MP than in PC (Figure 2e). The significant changes were observed in T+MP, with three mice exhibiting $(++)$ urine protein and eight mice with (+) urine protein.

Tofacitinib delayed the nephritic physiological progression of lupus mice. Diffuse proliferation, glomerular balloon adhesion, and solitary or multiple glomeruloscleroses were found in PC. After therapy, moderate multifocal hyperplasia, mild hyperplasia, or occasionally normal conditions were observed in $\mathrm{T}$ and $\mathrm{MP}$ with (a)
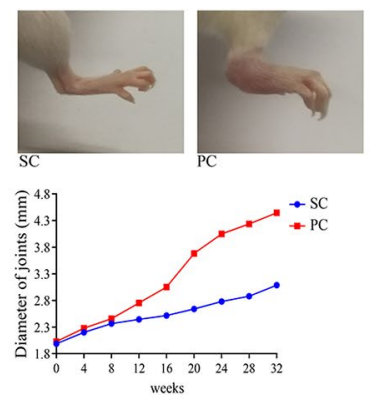

(b)
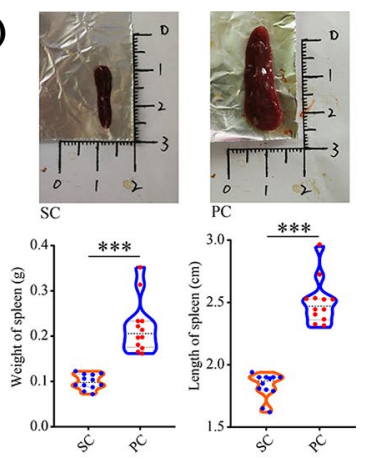

(c)
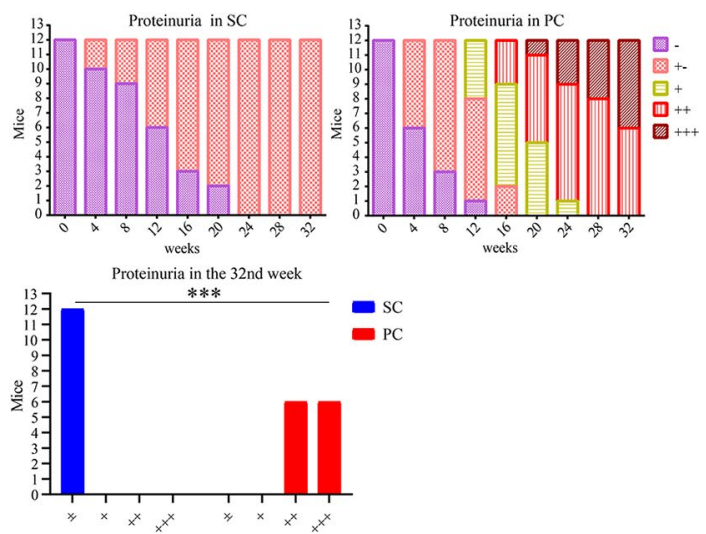

(f)
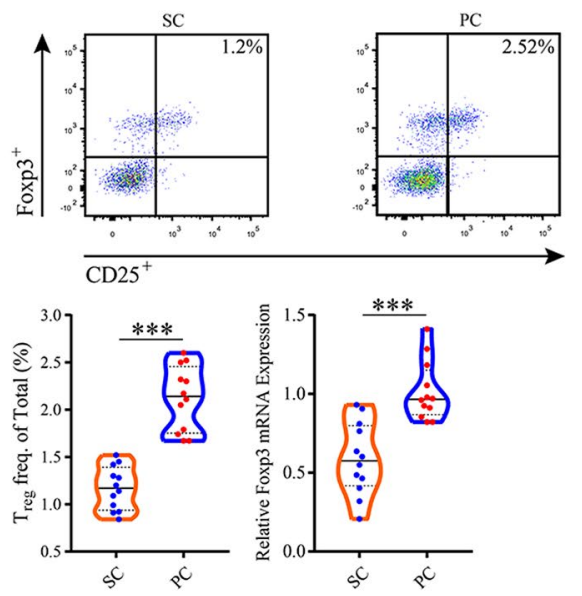

Figure 1. Pristane-induced murine model of lupus. Differences in joint length (a), spleen weight and length (b), and proteinuria (c) in SC and PC. Proteinuria concentration in PC at week 32 was significantly higher than that in SC $(p<0.001)$ (c). White punctate substances and fatty granuloma induced by pristane in PC were found inside abdominal cavity. Calcification of pristane at center of fatty granuloma with adipocytes and inflammatory cells around was observed via HE staining (d). Compared with SC, PC showed increased glomerulus volume, diffuse mesangial cell proliferation, and glomerular balloon adhesion with higher glomerular score, as indicated by HE staining (e). Compared with SC, PC had more hyperplasia of mesangial matrix and coil-like lesions in glomerulus, as shown by PAS staining (e). Percentages of splenic CD4+CD25+Foxp3+Treg cells were significantly higher in PC than in SC, and splenic Foxp3 mRNA expression was higher in PC than in SC (f). Compared with SC, PC presented a significantly higher serum concentration of IFN- $\gamma$, IL-6, and anti-dsDNA antibody (g).

CD: Cluster of differentiation; dsDNA: Double-stranded deoxyribonucleic acid; Foxp3: Forkhead box P3; HE: Hematoxylin-eosin; IFN- $\gamma$ : Interferon gamma; IL: Interleukin; mRNA: Messenger ribonucleic acid; ns: Not significant; PAS: Periodic acid-Schiff; PC: Pristane control; SC: Saline control; Treg: Regulatory T cells; ${ }^{*} \mathrm{p}<0.05 ;{ }^{* *} \mathrm{p}<0.01 ;{ }^{* * *} \mathrm{p}<0.001$. 
few glomeruli demonstrating diffuse proliferation, glomerular balloon adhesion, and no visible focal sclerosis. In T+MP, a small amount of normal glomeruli, less diffuse proliferation, less balloon adhesion, moderate multifocal hyperplasia, and mild focal hyperplasia were observed without focal segmental sclerosis (Figure 3a). All renal pathological scores decreased after treatment, with the greatest decline observed in $\mathrm{T}+\mathrm{MP}$, thereby indicating that the best curative effect was achieved (Figure 3b).

Apart from local sclerosis, hyperplasia of mesangial matrix was also observed around glomerular mesangial cells in PC. Hyperplasia of mesangial matrix was diminished in $\mathrm{T}, \mathrm{MP}$, and $\mathrm{T}+\mathrm{MP}$ (Figure 3c).

Tofacitinib decreased serum concentrations of anti-dsDNA antibody, IFN- $\gamma$, and IL- 6 in lupus mice. The serum concentrations of anti-dsDNA antibody, IFN- $\gamma$, and IL- 6 increased considerably in PC and decreased significantly in T, T+MP, and $\mathrm{MP}$ at varying degrees, with the greatest decline observed in T+MP after treatment (Figure 4a).

Tofacitinib decreased the percentages of splenic $T_{\text {reg }}$ cells in lupus mice. The percentages of splenic $\mathrm{T}_{\text {reg }}$ cells in lupus mice increased significantly in PC (Figure 1f) and decreased in the treatment groups after therapy. The most evident change was observed in $\mathrm{T}+\mathrm{MP}$, and the level of Foxp3 mRNA exhibited a similar tendency (Figure 4b).

Tofacitinib affected the mRNA expression of relevant genes downstream of the JAK-STAT pathway. Twelve relevant genes downstream of the JAK-STAT pathway were tested at the (a)

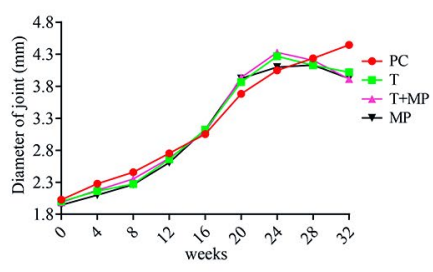

(b)

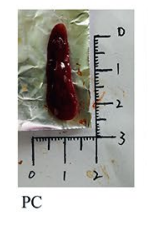

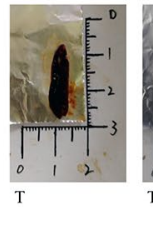

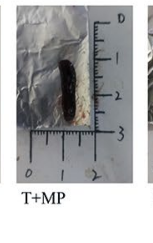

(c)

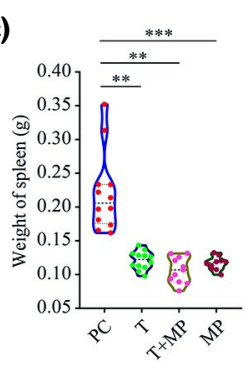

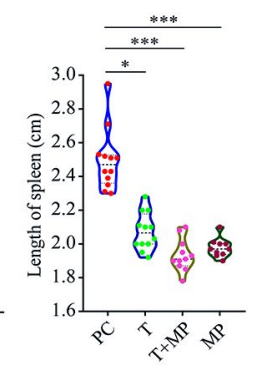

(d)
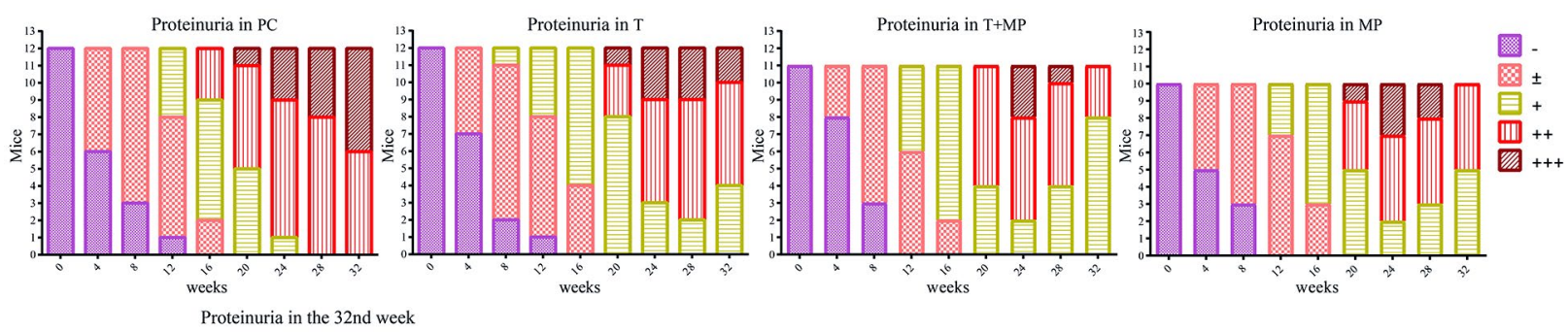

(e)

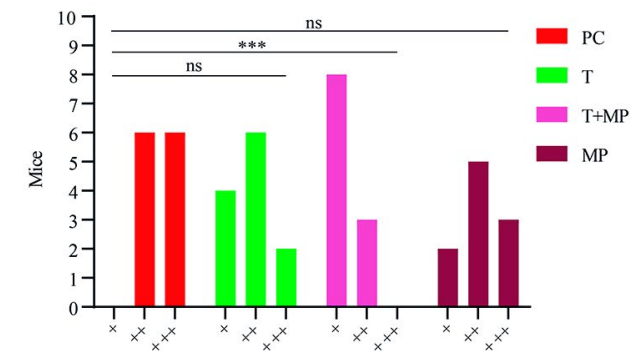

Figure 2. Relieved red swollen joints and splenomegaly and decreased urine protein level in lupus mice after therapy. (a) Change trends of joint diameters and (b and $\mathbf{c )}$ comparisons of spleen weight and length in all induced groups. (d) Change in urine protein levels in PC, T, T+MP, and MP. (e) At week 32, urine protein was reduced in T, T+MP, and MP than in PC.

MP: Methylprednisolone; ns: Not significant; PC: Pristane control; T: Tofacitinib; T+MP: Tofacitinib plus methylprednisolone; ${ }^{*} \mathrm{p}<0.05 ;{ }^{* *} \mathrm{p}<0.01$; ${ }^{* * * *} \mathrm{p}<0.001$. 
(a)

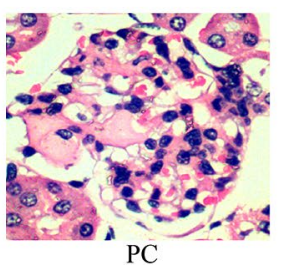

(c)

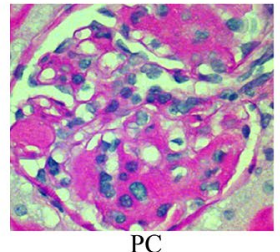

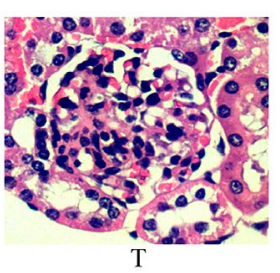

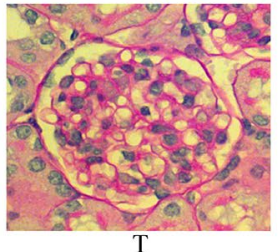

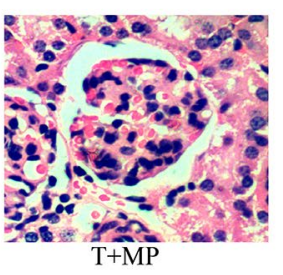

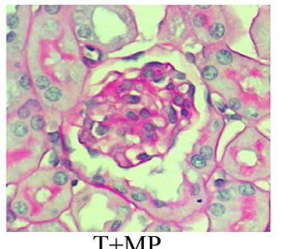

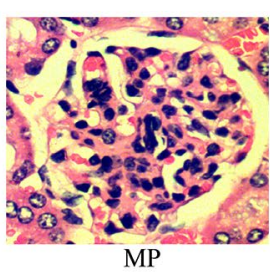

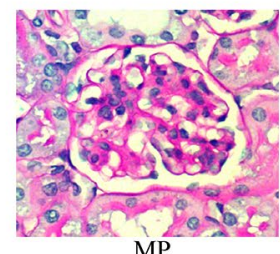

(b)

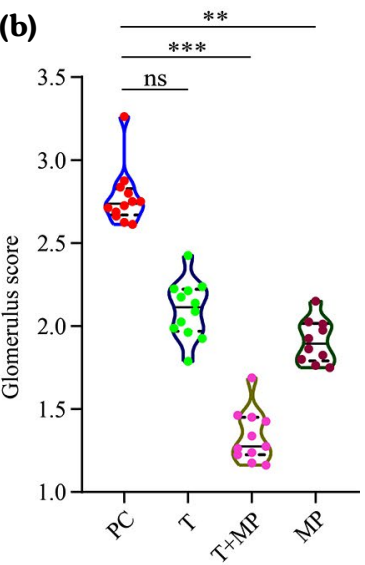

Figure 3. Renal pathological changes after therapy. (a) Renal pathological changes in PC, T, MP, and T+MP via H-E staining. (b) All quantification scores decreased after treatment and declined the most in T+MP. (c) PAS staining showed considerably less hyperplasia of mesangial matrix in T, MP, and T+MP than in PC.

H-E: Hematoxylin-eosin; MP: Methylprednisolone; ns: Not significant; PAS: Periodic acid-Schiff; PC: Pristane control; T: Tofacitinib; T+MP: Tofacitinib plus methylprednisolone; ${ }^{*} \mathrm{p}<0.05 ;{ }^{* *} \mathrm{p}<0.01 ;{ }^{* * *} \mathrm{p}<0.001$

(a)
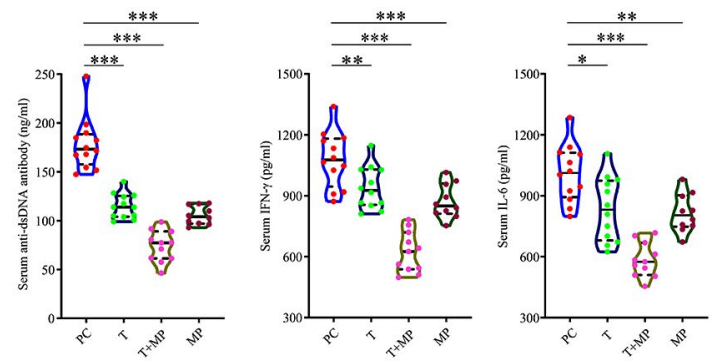

(b)
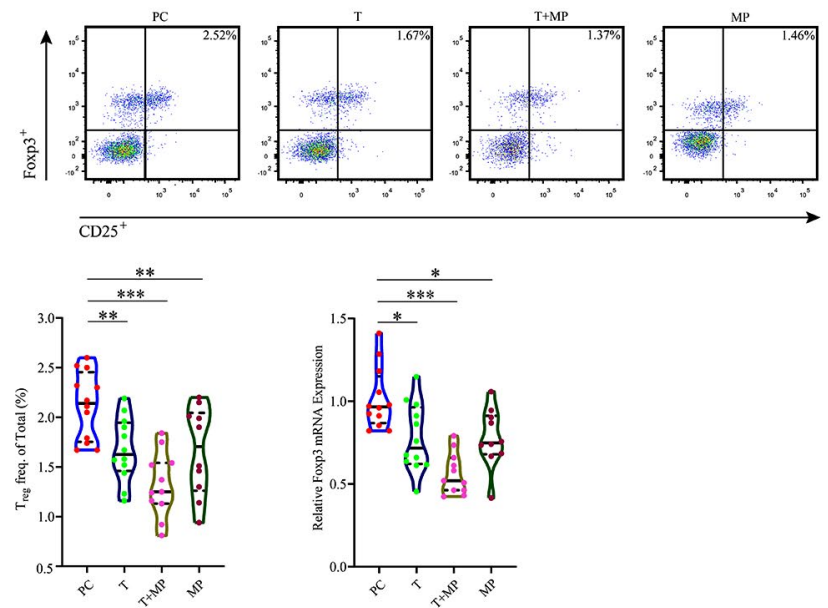

(c)
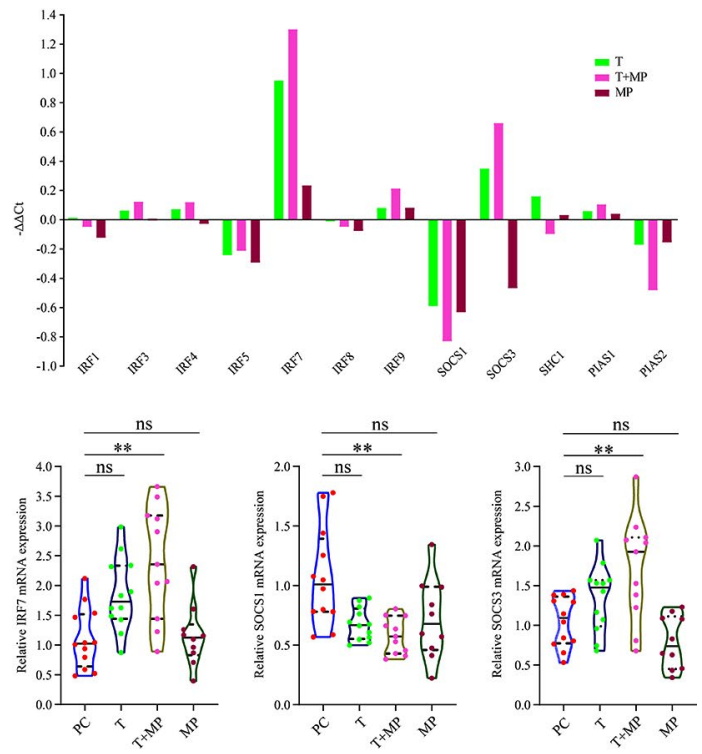

Figure 4. Effects of tofacitinib on lupus mice with regard to anti-double-stranded deoxyribonucleic acid antibody, interferon gamma, interleukin-6, splenic regulatory $\mathrm{T}$ cells, and messenger ribonucleic acid expression of relevant genes downstream of Janus kinase-signal transducer and activator of transcription pathway. (a) Compared with PC, T, MP, and T+MP presented a significantly lower serum concentration of anti-dsDNA antibody, IFN- $\gamma$, and IL-6. (b) Compared with PC, T, $\mathrm{MP}$, and $\mathrm{T}+\mathrm{MP}$ presented a significantly lower percentage of splenic $\mathrm{CD} 4^{+} \mathrm{CD} 25^{+} \mathrm{Foxp} 3^{+} \mathrm{T}_{\text {reg }}$ cells and splenic Foxp3 mRNA expression. (c) Compared with PC, expression levels of 12 relevant genes downstream of the JAK-STAT pathway in T, $\mathrm{MP}$, and T+MP were shown. Positive values represent higher levels than those in PC, and vice versa.

CD: Cluster of differentiation; dsDNA: Double-stranded deoxyribonucleic acid; Foxp3: Forkhead box P3; HE: Hematoxylin-eosin; IFN- $\gamma$ : Interferon gamma; JAK-STAT: Janus kinase-signal transducer and activator of transcription; IL: Interleukin; MP: Methylprednisolone; mRNA: Messenger ribonucleic acid; ns: Not significant; PAS: Periodic acid-Schiff; PC: Pristane control; SC: Saline control; T: Tofacitinib; T+MP: Tofacitinib plus methylprednisolone; Treg: Regulatory T cells; ${ }^{*} \mathrm{p}<0.05 ;{ }^{* *} \mathrm{p}<0.01 ;{ }^{* * *} \mathrm{p}<0.001$. 
mRNA level in the induced groups. The mRNA of several genes (interferon regulatory factor [IRF] 1, IRF3, IRF4, IRF5, IRF8, IRF9, Src homology 2 domain-containing, transforming protein 1, protein inhibitor of activated STAT [PIAS] 1, and PIAS2) did not present a statistical difference between PC and the treatment groups (Figure 4c). The IRF7 level was higher in T+MP $(p<0.01)$ and slightly higher in $T(p>0.05)$ and MP ( $>0.05)$ than in PC after treatment. The expression of suppressor of cytokine signaling (SOCS) 1 was lower in $\mathrm{T}$ ( $p>0.05), \mathrm{T}+\mathrm{MP}$ $(p<0.01)$, and MP $(p>0.05)$ than in PC. The SOCS3 level was higher in T $(p>0.05)$ and $T+M P$ $(p<0.01)$ and lower in MP $(p>0.05)$ than in PC (Figure 4c).

\section{DISCUSSION}

Systemic lupus erythematosus is a typical AID with a complicated mechanism. ${ }^{12}$ The conventional treatment for SLE primarily includes glucocorticoids and immunosuppressive agents, which often have significant side effects. Tofacitinib, a drug that preferentially targets JAK1 and JAK3, exerts pleiotropic beneficial effects in murine lupus. ${ }^{13}$ New Zealand black/ New Zealand white (NZB/NZW) F1 mice and Murphy Roths Large lymphoproliferative (MRL/lpr) mice are lupus prone. NZB/NZW F1 mice possess the defect of thymus, whereas MRL/lpr mice have a loss-of-function lpr mutation within the gene encoding Fas. ${ }^{14,15}$ $\mathrm{BALB} / \mathrm{c}$ mice can be induced to develop into immunocomplex glomerulonephritis with autoantibodies by pristane. ${ }^{16}$ Although we used $\mathrm{BALB} / \mathrm{c}$ mice for a pristane-induced lupus model other than SLE-prone mice, NZB/NZW F1 and MRL/lpr, which Furumoto et al. ${ }^{10}$ or Ikeda et al. ${ }^{13}$ used, we had consistent findings of reduced proinflammatory cytokine levels and amelioration of lupus nephritis, arthritis, and splenomegaly after treatment with tofacitinib. Different from Furumoto et al.'s ${ }^{10}$ study, the present work also explored the relationship of tofacitinib with murine $T_{\text {reg }}$ cells and 12 related genes downstream of the JAK-STAT signaling pathway. Although we analyzed spleen tissues herein whereas kidney tissues were analyzed in Ikeda et al.'s ${ }^{13}$ study, we had similar findings of significantly reduced frequency of $\mathrm{T}_{\text {reg }}$ cells and reduced Foxp3 expression in tofacitinibtreated mice. Tofacitinib modulated the SOCS in this study and modulated the genes related to the IFN-signaling pathway in the research of Furumoto et al. ${ }^{10}$ and Ikeda et al. ${ }^{13}$

The Foxp3 expression and splenic Foxp3+ $\mathrm{T}_{\text {reg }}$ cell percentage in $\mathrm{PC}$ were higher than those in $\mathrm{SC}$. These findings may be a response of $\mathrm{T}_{\text {reg }}$ to overwhelming inflammation, ${ }^{17}$ but the underlying mechanism has not been yet explained.

After treatment, T, MP, and T+MP showed a decline in inflamed joint diameters, urinary protein concentration, pathological score, and serum concentration of anti-dsDNA antibody, accompanied with decreased percentages of splenic $\mathrm{T}_{\text {reg }}$ cells. The observed decrease in $T_{\text {reg }}$ cell percentage may be a recovery indicator of lupus nephritis, apart from renal pathological changes and the serum concentration of anti-dsDNA antibody. Increased $\mathrm{T}_{\text {reg }}$ cell percentage may be a response to overwhelming inflammation, ${ }^{18}$ and a decrease with concurrently reduced Foxp3 expression may indicate amelioration after treatment. Furthermore, the decreased concentrations of IL- 6 and IFN- $\gamma$ indicated declined levels of inflammatory mediators and pro-inflammatory factors in vivo. Thus, decreased $\mathrm{T}_{\text {reg }}$ cell percentage may be used as a reference indicator of reduction of inflammation, symptom relief, and drug efficacy. Patients with SLE, particularly in early onset, have high IL-6 levels, which are related to disease activities. ${ }^{19,20}$ In the current study, the joint diameter, urine protein level, serum concentration of anti-dsDNA antibody, and $\mathrm{T}_{\text {reg }}$ cell percentage decreased the most in $\mathrm{T}+\mathrm{MP}$, with declining serum concentrations of IL- 6 and IFN- $\gamma$. The best recovery of lupus nephritis also occurred in T+MP according to the presented pathological scores. Thus, tofacitinib may be useful for lupus nephritis mice as it can ameliorate systemic inflammation, reduce the secretion of pro-inflammatory cytokines, and relieve symptoms. The curative effect of tofacitinib could be enhanced when combined with methylprednisolone. We found that the pathological score, the serum concentrations of anti-dsDNA antibody, IFN- $\gamma$, and IL- 6 , and the $\mathrm{T}_{\text {reg }}$ cell percentages may be appropriate indicators for detecting lupus nephritis. ${ }^{21}$ 
Tofacitinib can block the signal transduction pathway by inhibiting the phosphorylation of JAK-STAT. ${ }^{22-25}$ SLE and STAT are closely related. ${ }^{26,27}$ To identify the potential regulatory gene loci for tofacitinib, the present study tested 12 relevant genes downstream of the JAK-STAT pathway. Statistical differences were found in the mRNA expression levels of IRF7, as well as SOCS1 and SOCS3, but none were found in the other genes. IRF7 can promote the production of type 1 IFN, and a high expression of Foxp3 suppresses the positive feedback of IRF7 on type 1 IFN, thereby relieving inflammation caused by viral infection. ${ }^{28}$ In the current study, we speculated that the high IRF7 expression in PC may be related to the high level of type 1 IFN pro-inflammatory cytokines in mice with lupus nephritis, consistent with reports of significantly increased type 1 IFN level in patients with SLE. ${ }^{29}$ Type 1 IFN may also be induced by SOCS1 or through other mechanisms when IRF7 increases. ${ }^{30,31}$ However, herein, IRF7 did not decrease but increased evidently at the mRNA level with a decline of SOCS1 after treatment in $T$ and $\mathrm{T}+\mathrm{MP}$. We hypothesized that the antiinflammatory response was incomplete, and that the decrease in type 1 IFN expression remained limited even after treatment. This phenomenon could explain why complete recovery from lupus nephritis did not occur even though indicators improved (e.g., IFN- $\gamma$, pathological score, serum concentrations of anti-dsDNA antibody and IL-6, and $\mathrm{T}_{\text {reg }}$ cell percentage). This finding may be attributed to the effect of tofacitinib on decreasing SOCS1 expression through the JAK-STAT pathway, thereby limiting the inhibition effect of SOCS1 on IRF7. Consequently, the synthesis and release of type 1 IFN are promoted. However, type 1 IFNs such as IFN- $\gamma$ and IFN- $\gamma$ were not examined in the present study; as such, internal correlation could not be illustrated.

A SOCS is a negative regulator of the JAK-STAT pathway and can restrain cytokine signal transduction when combined with JAK. ${ }^{32,33}$ SOCS3 can also directly repress JAK1, JAK2, and tyrosine kinase 2 (Tyk2) but not JAK3. ${ }^{34}$ In the current study, SOCS3 increased with decreased IL-6 in T and T+MP. Tofacitinib can directly suppress JAK1 and JAK3 but maintain
JAK2 and Tyk2 within limits. ${ }^{35-37}$ Accordingly, we speculated that tofacitinib could expand the inhibitory signal conduction cascade reaction and ameliorate systemic inflammation by inducing SOCS3, which may thus be a potential therapeutic target in the future.

In patients with SLE, the expression of IRF-related genes (IRF7, IFN-stimulated gene [ISG] 15, and IFN-inducible transmembrane protein 1) significantly increases during the active stage. ${ }^{38}$ Furthermore, type 1 IFN regulatory genes in immature $\mathrm{CD}^{+} \mathrm{T}$ cells are hypomethylated and overexpressed. ${ }^{39}$ ISG15 is a potential bridge between types I and II IFN immune responses. ${ }^{40}$ In the current study, IRF7 changed after tofacitinib administration. Thus, IFN regulatory genes may be potential pathogenic genes that regulate IFN levels and promote systemic inflammation and may serve as a therapeutic target for tofacitinib. We believe that tofacitinib is useful for lupus nephritis but cannot completely cure it even when combined with methylprednisolone. Drugs for decreasing type 1 IFN cytokine are possibly required.

The limitations of this study were as follows: functional examination of $\mathrm{T}_{\text {reg }}$ cells was lacking, and the result of proteinuria analysis would have been better if ELISA was used instead of test papers.

In conclusion, tofacitinib can reduce the cytokine levels of IL- 6 and IFN- $\gamma$ and the level of anti-dsDNA, ameliorate systemic inflammation, and relieve nephritis and arthritis in a pristaneinduced murine model of lupus. Tofacitinib and methylprednisolone may act synergistically in the treatment of lupus in mice. SOCS3 may be involved during tofacitinib treatment.

Ethics Committee Approval: Animal use protocols were approved by the Animal Experimental Ethical Inspection Committee of Fujian Medical University (ethical no: FJMU IACUC 2020-0023). The study was conducted in accordance with the principles of the Declaration of Helsinki.

Data Sharing Statement: The data that support the findings of this study are available from the corresponding author upon reasonable request.

Author Contributions: Performed the experiments: J.L., Y.Z., M.W., Y.Z., P.L.; Designed and interpreted the data: Y.C.; Designed and performed the experiments, interpreted the data, and wrote the manuscript: X.Y. 
Conflict of Interest: The authors declared no conflicts of interest with respect to the authorship and/or publication of this article.

Funding: This work was supported by grants from Fujian Provincial Natural Science Foundation (Grant no. 2016J01551), Fujian Provincial Health Project (Grant no. 2018-CX-19), Fujian Provincial Health Project (Grant no.2017-CX-20) and Fujian Province Joint Funds for the Innovation of Science and Technology (Grant no. 2017Y9051).

\section{REFERENCES}

1. Mohan C, Putterman C. Genetics and pathogenesis of systemic lupus erythematosus and lupus nephritis. Nat Rev Nephrol 2015;11:329-41.

2. Goropevsek A, Holcar M, Avcin T. The Role of STAT signaling pathways in the pathogenesis of systemic lupus erythematosus. Clin Rev Allergy Immunol 2017;52:164-81.

3. Ramírez-Vélez G, Medina F, Ramírez-Montaño L, Zarazúa-Lozada A, Hernández R, Llorente L, et al. Constitutive phosphorylation of interferon receptor A-associated signaling proteins in systemic lupus erythematosus. PLoS One 2012;7:e41414.

4. Ivashkiv LB, Donlin LT. Regulation of type I interferon responses. Nat Rev Immunol 2014;14:36-49.

5. Harada T, Kyttaris V, Li Y, Juang YT, Wang Y, Tsokos GC. Increased expression of STAT3 in SLE T cells contributes to enhanced chemokine-mediated cell migration. Autoimmunity 2007;40:1-8.

6. Durant L, WatfordWT, Ramos HL, Laurence A, Vahedi $\mathrm{G}$, Wei L, et al. Diverse targets of the transcription factor STAT3 contribute to T cell pathogenicity and homeostasis. Immunity 2010;32:605-15.

7. Goropevsek A, Gorenjak M, Gradisnik S, Dai K, Holc I, Hojs R, et al. STAT5 phosphorylation in CD4 T cells from patients with SLE is related to changes in their subsets and follow-up disease severity. $\mathrm{J}$ Leukoc Biol 2017;101:1405-18.

8. Malemud CJ. The role of the JAK/STAT signal pathway in rheumatoid arthritis. Ther Adv Musculoskelet Dis 2018;10:117-27.

9. Wallace DJ, Furie RA, Tanaka Y, Kalunian KC, Mosca M, Petri MA, et al. Baricitinib for systemic lupus erythematosus: a double-blind, randomised, placebo-controlled, phase 2 trial. Lancet 2018;392:222-31.

10. Furumoto Y, Smith CK, Blanco L, Zhao W, Brooks SR, Thacker SG, et al. Tofacitinib Ameliorates Murine Lupus and Its Associated Vascular Dysfunction. Arthritis Rheumatol 2017;69:148-60.

11. Tao X, Fan F, Hoffmann V, Longo NS, Lipsky PE. Therapeutic impact of the ethyl acetate extract of Tripterygium wilfordii Hook F on nephritis in NZB/W F1 mice. Arthritis Res Ther 2006;8:R24.
12. Sekigawa I, Naito T, Hira K, Mitsuishi K, Ogasawara $\mathrm{H}$, Hashimoto $\mathrm{H}$, et al. Possible mechanisms of gender bias in SLE: a new hypothesis involving a comparison of SLE with atopy. Lupus 2004;13:217-22.

13. Ikeda K, Hayakawa K, Fujishiro M, Kawasaki M, Hirai $\mathrm{T}$, Tsushima $\mathrm{H}$, et al. JAK inhibitor has the amelioration effect in lupus-prone mice: the involvement of IFN signature gene downregulation. BMC Immunol 2017;18:41.

14. Taguchi N, Hashimoto Y, Naiki M, Farr AG, Boyd RL, Ansari AA, et al. Abnormal thymic expression of epithelial cell adhesion molecule (EP-CAM) in New Zealand Black (NZB) mice. J Autoimmun 1999;13:393-404.

15. Watanabe-Fukunaga R, Brannan CI, Copeland NG, Jenkins NA, Nagata S. Lymphoproliferation disorder in mice explained by defects in Fas antigen that mediates apoptosis. Nature 1992;356:314-7.

16. Satoh M, Kumar A, Kanwar YS, Reeves WH. Anti-nuclear antibody production and immunecomplex glomerulonephritis in BALB/c mice treated with pristane. Proc Natl Acad Sci U S A 1995;92:10934-8.

17. Abe J, Ueha S, Suzuki J, Tokano Y, Matsushima K, Ishikawa S. Increased Foxp3(+) CD4(+) regulatory $\mathrm{T}$ cells with intact suppressive activity but altered cellular localization in murine lupus. Am $\mathrm{J}$ Pathol 2008;173:1682-92.

18. Zecevic L, Karamehic J, Coric J, Stubljar D, Avdagic N, Selmanovic K, et al. Potential immune biomarkers in diagnosis and clinical management for systemic lupus erythematosus. J Med Biochem 2018;37:163-71.

19. Munroe ME, Vista ES, Guthridge JM, Thompson LF, Merrill JT, James JA. Proinflammatory adaptive cytokine and shed tumor necrosis factor receptor levels are elevated preceding systemic lupus erythematosus disease flare. Arthritis Rheumatol 2014;66:1888-99.

20. Talaat RM, Mohamed SF, Bassyouni IH, Raouf AA. Th1/Th2/Th17/Treg cytokine imbalance in systemic lupus erythematosus (SLE) patients: Correlation with disease activity. Cytokine 2015;72:146-53.

21. Su DL, Lu ZM, Shen MN, Li X, Sun LY. Roles of proand anti-inflammatory cytokines in the pathogenesis of SLE. J Biomed Biotechnol 2012;2012:347141.

22. O'Shea JJ, Holland SM, Staudt LM. JAKs and STATs in immunity, immunodeficiency, and cancer. N Engl J Med 2013;368:161-70.

23. Rizzi M, Lorenzetti R, Fischer K, Staniek J, Janowska I, Troilo A, et al. Impact of tofacitinib treatment on human B-cells in vitro and in vivo. J Autoimmun 2017;77:55-66.

24. Baker KF, Isaacs JD. Novel therapies for immunemediated inflammatory diseases: What can we learn from their use in rheumatoid arthritis, spondyloarthritis, systemic lupus erythematosus, psoriasis, Crohn's disease and ulcerative colitis? Ann Rheum Dis 2018;77:175-87. 
25. Robinette ML, Cella M, Telliez JB, Ulland TK, Barrow AD, Capuder K, et al. Jak3 deficiency blocks innate lymphoid cell development. Mucosal Immunol 2018;11:50-60.

26. Deenick EK, Avery DT, Chan A, Berglund LJ, Ives ML, Moens L, et al. Naive and memory human B cells have distinct requirements for STAT3 activation to differentiate into antibody-secreting plasma cells. J Exp Med 2013;210:2739-53.

27. Yiu G, Rasmussen TK, Ajami B, Haddon DJ, Chu AD, Tangsombatvisit S, et al. Development of Th17Associated Interstitial Kidney Inflammation in LupusProne Mice Lacking the Gene Encoding STAT-1. Arthritis Rheumatol 2016;68:1233-44.

28. Litvak V, Ratushny AV, Lampano AE, Schmitz F, Huang AC, Raman A, et al. A FOXO3-IRF7 gene regulatory circuit limits inflammatory sequelae of antiviral responses. Nature 2012;490:421-5.

29. Rezaei R, Mahmoudi M, Gharibdoost F, Kavosi H, Dashti N, Imeni V, et al. IRF7 gene expression profile and methylation of its promoter region in patients with systemic sclerosis. Int J Rheum Dis 2017;20:1551-61.

30. Yu X, Cai B, Wang M, Tan P, Ding X, Wu J, et al. Cross-regulation of two type I interferon signaling pathways in plasmacytoid dendritic cells controls anti-malaria immunity and host mortality. Immunity 2016;45:1093-107.

31. Yu X, Du Y, Cai C, Cai B, Zhu M, Xing C, et al. Inflammasome activation negatively regulates MyD88IRF7 type I IFN signaling and anti-malaria immunity. Nat Commun 2018;9:4964.

32. Kershaw NJ, Murphy JM, Liau NP, Varghese LN, Laktyushin A, Whitlock EL, et al. SOCS3 binds specific receptor-JAK complexes to control cytokine signaling by direct kinase inhibition. Nat Struct Mol Biol 2013;20:469-76.
33. Yoshimura A, Ito M, Chikuma S, Akanuma T, Nakatsukasa $H$. Negative regulation of cytokine signaling in immunity. Cold Spring Harb Perspect Biol 2018;10:a028571.

34. Babon JJ, Kershaw NJ, Murphy JM, Varghese LN, Laktyushin A, Young SN, et al. Suppression of cytokine signaling by SOCS3: characterization of the mode of inhibition and the basis of its specificity. Immunity 2012;36:239-50.

35. Changelian PS, Flanagan ME, Ball DJ, Kent CR, Magnuson KS, Martin WH, et al. Prevention of organ allograft rejection by a specific Janus kinase 3 inhibitor. Science 2003;302:875-8.

36. Karaman MW, Herrgard S, Treiber DK, Gallant P, Atteridge CE, Campbell BT, et al. A quantitative analysis of kinase inhibitor selectivity. Nat Biotechnol 2008;26:127-32.

37. Meyer DM, Jesson MI, Li X, Elrick MM, FunckesShippy CL, Warner JD, et al. Anti-inflammatory activity and neutrophil reductions mediated by the JAK1/JAK3 inhibitor, CP-690,550, in rat adjuvantinduced arthritis. J Inflamm (Lond) 2010;7:41.

38. Kawasaki M, Sekigawa I, Nozawa K, Kaneko H, Takasaki Y, Takamori K, et al. Changes in the gene expression of peripheral blood mononuclear cells during the menstrual cycle of females is associated with a gender bias in the incidence of systemic lupus erythematosus. Clin Exp Rheumatol 2009;27:260-6.

39. Coit P, Jeffries M, Altorok N, Dozmorov MG, Koelsch KA, Wren JD, et al. Genome-wide DNA methylation study suggests epigenetic accessibility and transcriptional poising of interferon-regulated genes in naïve CD4+ T cells from lupus patients. J Autoimmun 2013;43:78-84.

40. Fan JB, Zhang DE. ISG15 regulates IFN- $\gamma$ immunity in human mycobacterial disease. Cell Res 2013;23:173-5. 